\title{
Dependence of the non-stationary form of Yaglom's equation on the Schmidt number
}

\author{
By P. ORLANDI AND R. A. ANTONIA ${ }^{2}$ \\ ${ }^{1}$ Departimento di Meccanica e Aeronautica, Universita Degli Studi di Roma 'La Sapienza', \\ 00184 Rome, Italy \\ ${ }^{2}$ Department of Mechanical Engineering, University of Newcastle, N.S.W. 2308, Australia
}

(Received 7 August 2001 and in revised form 26 September 2001)

The dynamic equation for the second-order moment of a passive scalar increment is investigated in the context of DNS data for decaying isotropic turbulence at several values of the Schmidt number $S c$, between 0.07 and 7 . When the terms of the equation are normalized using Kolmogorov and Batchelor scales, approximate independence from $S c$ is achieved at sufficiently small $r / \eta_{B}(r$ is the separation across which the increment is estimated and $\eta_{B}$ is the Batchelor length scale). The results imply approximate independence of the mixed velocity-scalar derivative skewness from $S c$ and underline the importance of the non-stationarity. At small $r / \eta_{B}$, the contribution from the non-stationarity increases as $S c$ increases.

\section{Introduction}

The transport equations for two-point correlations of longitudinal velocity and passive scalar fluctuations in isotropic turbulence were first written by Kármán \& Howarth (1938) and Corrsin (1951). They have been reproduced in a number of texts, e.g. Monin \& Yaglom (1975, equations 14.9 and 14.59). Adopting the notation used by the latter authors, these correlations are denoted by $B_{L L}(r, t)$ and $B_{\theta \theta}(r, t)$, where $r$ is the separation between the two points. The corresponding second-order structure functions are denoted by $D_{L L}(r, t)$ and $D_{\theta \theta}(r, t)$, where (for homogeneous turbulence)

$$
\frac{1}{2} D_{K K}(r, t)=B_{K K}(0, t)-B_{K K}(r, t),
$$

with $K$ standing for either $L$ or $\theta$. Third-order correlations are denoted by $B_{L L, L}$ and $B_{L \theta, \theta}$ and are related to the third-order structure functions via

$$
D_{L L L}(r, t)=6 B_{L L, L}(r, t), \quad D_{L \theta \theta}(r, t)=4 B_{L \theta, \theta}(r, t) .
$$

The transport equations for $D_{L L}$ and $D_{\theta \theta}$ were written by Kolmogorov (1941) and Yaglom (1949) respectively for stationary homogeneous isotropic turbulence at large Reynolds number:

$$
\begin{aligned}
& -D_{L L L}(r)+6 v \frac{\partial D_{L L}(r)}{\partial r}=\frac{4}{5}\langle\epsilon\rangle r, \\
& -D_{L \theta \theta}(r)+2 \kappa \frac{\partial D_{\theta \theta}(r)}{\partial r}=\frac{2}{3}\langle\chi\rangle r,
\end{aligned}
$$

where $\langle\epsilon\rangle$ and $\langle\chi\rangle$ are the dissipation rates of the turbulent kinetic energy $\left\langle q^{2}\right\rangle / 2$ and 
the scalar variance $\left\langle\theta^{2}\right\rangle$ respectively, defined by

$$
\begin{aligned}
& 2 v \int_{0}^{\infty} k^{2} E(k) \mathrm{d} k=\langle\epsilon\rangle, \\
& 2 \kappa \int_{0}^{\infty} k^{2} G(k) \mathrm{d} k=\langle\chi\rangle,
\end{aligned}
$$

where $E(k)$ and $G(k)$ are the three-dimensional energy and scalar spectra ( $v$ and $\kappa$ are the molecular and scalar diffusivities respectively). Note that (1.2) contains $2 / 3$ instead of $4 / 3$ in the usual form of Yaglom's equation when $\langle\chi\rangle$ is defined as the dissipation rate of $\left\langle\theta^{2}\right\rangle / 2$. The retention of the non-stationarity leads to the appearance of an additional term in either (1.1) or (1.2) (e.g. Saffman 1968; Lindborg 1999; Danaila et al. 1999; Hill 2001), namely

$$
-D_{L L L}(r, t)+6 v \frac{\partial D_{L L}(r, t)}{\partial r}-\frac{3}{r^{4}} \int_{0}^{r} s^{4} \frac{\partial D_{L L}(s, t)}{\partial t} \mathrm{~d} s=\frac{4}{5}\langle\epsilon\rangle r,
$$

and

$$
-D_{L \theta \theta}(r, t)+2 \kappa \frac{\partial D_{\theta \theta}(r, t)}{\partial r}-\frac{3}{r^{2}} \int_{0}^{r} s^{2} \frac{\partial D_{\theta \theta}(s, t)}{\partial t} \mathrm{~d} s=\frac{2}{3}\langle\chi\rangle r,
$$

where $s$ is a dummy variable representing the separation. Equations (1.5) and (1.6) can be symbolically rewritten as

$$
A+B+S=\frac{4}{5}\langle\epsilon\rangle r, \quad A_{\theta}+B_{\theta}+S_{\theta}=\frac{2}{3}\langle\chi\rangle r
$$

respectively. The new (integral) terms $S$ and $S_{\theta}$ can be interpreted as 'source' terms since they have the same sign as $A$ (or $A_{\theta}$ ) and $B$ (or $B_{\theta}$ ). The contribution of $S$ in (1.5) has been shown to be significant, at least at moderate values of the Reynolds number, for values of $r$ which would normally be identified with the inertial range (Danaila et al. 1999; Lindborg 1999; Alvelius 1999; Alvelius \& Johansson 2000). Alvelius (1999) used forcing in a large-eddy simulation to generate statistically stationary homogeneous turbulence; in this way, the Kolmogorov ' $4 / 5$ ' relation was more or less recovered, in contrast with a simulation of decaying turbulence for which the peak value of $D_{L L L} /(r\langle\epsilon\rangle)$ is only about 0.5 . Danaila et al. (1999) also showed that $S_{\theta}$ is equally important for slightly heated grid turbulence at a low Reynolds number and one value $(\simeq 0.7)$ of the Prandtl number $\operatorname{Pr}(\equiv v / \kappa$, where $\kappa$ is the thermal diffusivity of the fluid). In Danaila et al. (1999) the Eulerian time derivative $\partial / \partial t$ in (1.5) and (1.6) is strictly zero in the laboratory reference frame but non-zero in a system moving with the mean flow since the turbulence decays along the mean flow direction. In the present work, the turbulence is homogeneous but decays with respect to time. The present paper essentially extends the investigation of Danaila et al. (1999) by assessing the relative importance of the non-stationary term when the Schmidt number $S c$ ( $\equiv v / \kappa$, where $\kappa$ is the molecular diffusivity of the scalar) varies significantly. Direct numerical simulation (DNS) data for decaying homogeneous (and nearly isotropic) turbulence with $S c$ equal to $0.07,0.3,0.7,1,3$ and 7 are used to test normalized forms of (1.5) and (1.6). The normalization is discussed in $\S 2$ while brief details on the simulations are given in $\S 3$. The results are presented and discussed in $\S 4$. Part of the motivation for this paper is the need to re-interpret the existing framework of small-scale phenomenology (e.g. Warhaft 2000) when effects extraneous to (1.1) and (1.2) are present. 


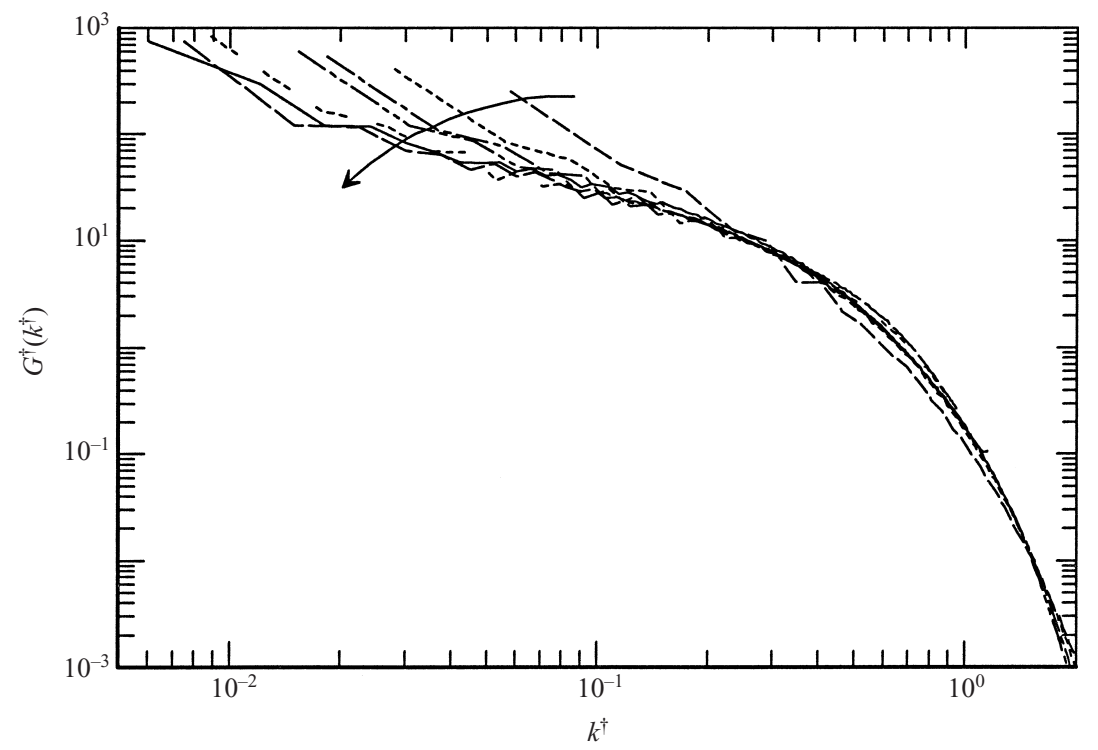

FIGURE 1. Three-dimensional scalar spectra at all values of $S c .--, S c=0.07 ;---, 0.3 ;---, 0.7$; $--\ldots, 1 ;--\ldots \quad--, 3 ;-\ldots, 7$. The solid curve was obtained from a pseudo-spectral simulation at $S c=7$. The arrow is in the direction of increasing $S c$.

\section{Normalization of generalized equations}

The Kolmogorov velocity $\left(U_{K} \equiv v^{1 / 4}\langle\epsilon\rangle^{1 / 4}\right)$ and length $\left(\eta \equiv v^{3 / 4}\langle\epsilon\rangle^{-1 / 4}\right)$ scales are appropriate for normalizing (1.5) when the focus is on small scales, i.e. for values of $r$ in the dissipative and inertial ranges. The Kolmogorov-normalized form of (1.5) is

$$
A^{*}+B^{*}+S^{*}=\frac{4}{5} r^{*},
$$

where the asterisk denotes Kolmogorov normalization. For the present range of $S c$, Batchelor length $\eta_{B}\left(\equiv \eta S c^{-1 / 2}\right)$ and scalar $\theta_{B}\left(\equiv\left[\langle\chi\rangle \eta / U_{K}\right]^{1 / 2}\right)$ scales seem appropriate for normalizing small-scale scalar quantities. It has now been established (e.g. Kerr 1990; Gibson 1991; Pumir 1994; Bogucki, Domaradzki \& Yeung 1997; Brethouwer \& Nieuwstadt 1999; Nieuwstadt \& Brethouwer 2000; Yeung, Sykes \& Vedula 2000) that the high-wavenumber portion of the scalar spectrum (or correspondingly the scalar structure function at small separations) scales on $\eta_{B}$ and $\theta_{B}$, at least when $S c$ is greater than about 0.1. The present distributions of $G^{\dagger}\left(k^{\dagger}\right)$ (the dagger denotes normalization by $\eta_{B}$ and $/$ or $\theta_{B}$ ) in figure 1 indicate a satisfactory collapse for $k^{\dagger} \gtrsim 0.2$ for all values of $S c$ except $S c=0.07$ (a possible difficulty here is the very small value of $P e_{\lambda_{\theta}}$ in our simulation). The high-wavenumber collapse of $G^{\dagger}\left(k^{\dagger}\right)$ in figure 1 is also confirmed (the plot is not shown here) by the good collapse, for $S c \geqslant 0.3$, of the probability density functions of $D_{\theta \theta}^{\dagger}$ at sufficiently small $r^{\dagger}$. The collapse reflects the role of the compressive strain rate $\gamma \equiv(\langle\epsilon\rangle / v)^{1 / 2}$ (note that $\eta_{B} \equiv(\kappa / \gamma)^{1 / 2}$ ) in the formation of scalar sheets. Batchelor (1959) showed that $\gamma$ is the relevant parameter for mixing at the smallest scales when $S c \gg 1$. However, Gibson $(1968 a, b)$ argued that $\gamma$ is relevant to the small-scale mixing process irrespectively of $S c$. For $S c \ll 1$, Batchelor, Howells $\&$ Townsend (1959) assumed that the Obukhov-Corrsin scale $\eta_{O C} \equiv\left(\kappa^{3} /\langle\epsilon\rangle\right)^{1 / 4}$ was relevant and proposed that $G(k) \sim k^{-17 / 3}$ when $\eta_{O C}^{-1}<k<\eta^{-1}$. Gibson $(1968 b)$ also predicted a $k^{-17 / 3}$ behaviour in the range $\eta_{B}^{-1}<k<\eta^{-1}$ and a $k^{-3}$ behaviour for 
$\eta_{O C}^{-1}<k<\eta_{B}^{-1}$. Available evidence, based either on experimental or numerical data, does not however seem to support either of these two results conclusively.

Included in figure 1 is the distribution of $G(k)$ at $S c=7$ obtained with a finer resolution $\left(384^{3}\right)$ pseudo-spectral simulation; it is in reasonable agreement with the present finite difference distributions. The ratio $\left(\eta / U_{K}\right)$, or Kolmogorov time scale $t_{K}$, which appears in $\theta_{B}$ essentially reflects the action of the compressive strain rate $\left.(\langle\epsilon\rangle / v)^{1 / 2}\right)$ on the scalar. It is therefore appropriate to normalize $t$ in (1.6) in the same way as in (1.5). It is also appropriate to continue to use $U_{K}$ to normalize the velocity fluctuation contained in $D_{L L L}$. The normalized form of (1.6) can then be written as

$$
A_{\theta}^{\dagger}+B_{\theta}^{\dagger}+S_{\theta}^{\dagger}=\frac{2}{3} r^{\dagger},
$$

where $A_{\theta}^{\dagger} \equiv S c^{1 / 2} D_{L \theta \theta}^{\dagger}\left(r^{\dagger}, t^{*}\right)$. The appearance of $S c^{1 / 2}$ in $A_{\theta}$ suggests that, within this normalization framework (its relevance is tested in $\S 4$ ), dynamic similarity for the smallest scales requires that $S c$ should remain constant.

\section{Numerical simulations}

All the results presented here have been obtained with a finite difference scheme $\left(250^{3}\right.$ grid), which is second-order in space and time, on a desktop workstation. For a particular value of $S c$, a computational time of about 5 days was required for 10 computational time units, with a time step of 0.02 . The simulation was performed in a cubic box of size $2 \pi$ with a prescribed energy spectrum at $t=0$. The turbulent velocity field is homogeneous; there is however a slight anisotropy at large scales, e.g. $\left\langle v^{2}\right\rangle /\left\langle u^{2}\right\rangle \simeq 1.14$ and $\left\langle w^{2}\right\rangle /\left\langle u^{2}\right\rangle \simeq 1.13$, where $u, v, w$ are the velocity fluctuations in the $x$-, $y$-, $z$-directions respectively $\left(\left\langle q^{2}\right\rangle \equiv\left\langle u^{2}\right\rangle+\left\langle v^{2}\right\rangle+\left\langle w^{2}\right\rangle\right)$. For the scalar, the simulation was initiated with a random phase spectrum similar in shape to that of $\left\langle q^{2}\right\rangle$. A finite relaxation time was required before $\left\langle q^{2}\right\rangle$ and $\left\langle\theta^{2}\right\rangle$ displayed power-law decay rates. Velocity and passive scalar fields at $t=10$ (or $t^{*}=11.8$ ) were used as the initial conditions for the calculation of the subsequent 10 time units. At $t=20$, the estimate of $\langle\chi\rangle$ for $S c=7$ was found to be too low; for this case, the simulation was extended a further 10 time steps (i.e. to $t=30$ ) so as to allow satisfactory convergence of the integral in (1.4). More details on the simulation, including the effect of different initial conditions, are given in Orlandi \& Antonia (2001). At $t=20$ (or $t^{*} \simeq 23.6$ ), the Taylor microscale Reynolds number $R_{\lambda}$ was about $47\left(R_{\lambda}\right.$ decays only slowly between $t=20$ and 30). The turbulent Péclet number $P e_{\lambda_{\theta}} \equiv\left(\left\langle q^{2}\right\rangle / 3\right)^{1 / 2} \lambda_{\theta} / \kappa$ (the Corrsin microscale $\lambda_{\theta}$ is defined by the ratio $\left.\left\langle\theta^{2}\right\rangle^{1 / 2} /\left\langle(\partial \theta / \partial x)^{2}\right\rangle\right)$ varies as $S c^{1 / 2}$; it increases from 13.6 at $S c=0.07$ to 136 at $S c=7$. Spectra of velocity and scalar $(S c=0.7)$ fluctuations were in good agreement with those measured in grid turbulence at a comparable $R_{\lambda}$ (Zhou et al. 2000; Danaila et al. 2000).

Structure functions were evaluated in physical space. Although this is a timeconsuming calculation, it need only be done at one particular time within the range for which $\left\langle q^{2}\right\rangle$ and $\left\langle\theta^{2}\right\rangle$ exhibit power-law decay rates. It can be implemented more efficiently via FFTs, especially if a long-time evolution of $\partial D_{K K} / \partial t$ is required.

\section{Results}

We examine here the relative contributions of the terms in (2.1) and (2.2) evaluated at $t=20$ for $0.07 \leqslant S c \leqslant 3$ and $t=30$ for $S c=7$. Differentiation of $D_{L L}$ and $D_{\theta \theta}$ with respect to $r$ was relatively straightforward. The temporal derivatives of $D_{L L}$ and 


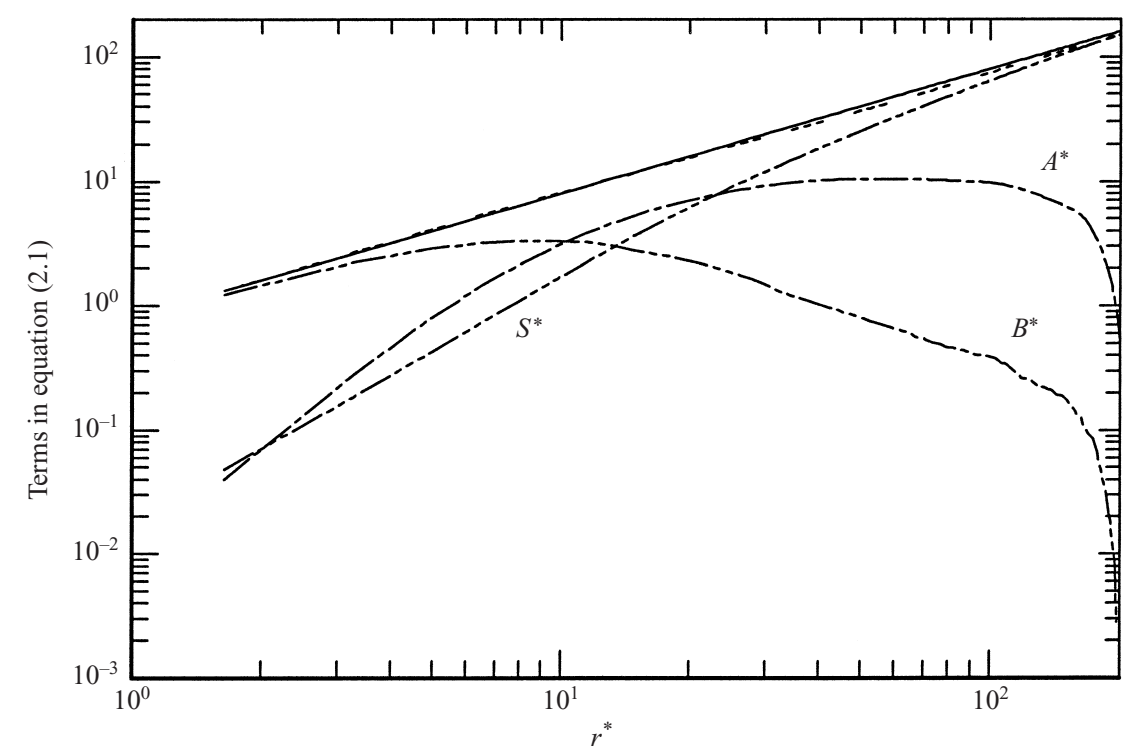

FIGURE 2. Estimates of all terms in the Kolmogorov-normalized equation (2.1), namely $A^{*}+B^{*}+S^{*}=(4 / 5) r^{*} .--\longrightarrow, A^{*} ;-\cdots, B^{*} ;-\cdots, S^{*} ;-,(4 / 5) r^{*} ;-\cdots_{-}, A^{*}+B^{*}+S^{*}$.

$D_{\theta \theta}$ were estimated from values of $D_{L L}$ and $D_{\theta \theta}$ stored at three consecutive times centred either at $t=20$ or 30 .

Estimates of $A^{*}, B^{*}, S^{*}$ are shown in figure 2 together with $(4 / 5) r^{*}$, i.e. the term on the right-hand side of (2.1). As expected, the viscous term $B^{*}$ provides the major contribution at small $r^{*}$. Term $A^{*}$ increases with $r^{*}$ and becomes comparable in magnitude to $B^{*}$ at $r^{*} \simeq 10$ (this location has been shown not to be significantly affected by the Reynolds number); at large enough $r^{*}$, this term must go to zero as the velocity fluctuations at the two points become uncorrelated. Term $S^{*}$, which reflects the non-stationarity of this flow, increases steadily with $r^{*}$, overtaking term $A$ at $r^{*}$ equal to about 20 (for reference, $\lambda^{*} \simeq 15$ ). At larger $r^{*}, S^{*}$ provides the major contribution to $(4 / 5) r^{*}$, approaching this latter value when $r^{*} \gtrsim 100$. This behaviour is expected since, at sufficiently large scales (for reference, $\left.L^{*} \simeq 124\right),(2.1)$ reduces to

$$
\langle\epsilon\rangle=-\frac{\mathrm{d}\left\langle q^{2}\right\rangle / 2}{\mathrm{~d} t}
$$

namely a balance between the energy dissipation rate and the kinetic energy decay rate. This point was discussed by Danaila et al. (1999) and Antonia et al. (2000). The latter also noted that, as $r^{*} \rightarrow 1,(2.1)$ correctly represents the behaviour of the transport equation of $\langle\epsilon\rangle$ or, for homogeneous turbulence, the enstrophy $\left\langle\omega^{2}\right\rangle$ (e.g. Batchelor \& Townsend 1947 ). The sum of $A^{*}, B^{*}$ and $S^{*}$ is also shown in figure 2. It is practically indistinguishable from $(4 / 5) r^{*}$; the imbalance $I$, or difference between $(4 / 5) r^{*}$ and $\left(A^{*}+B^{*}+S^{*}\right)$, is small (except at very small and very large $r^{*}$, the ratio $I /\left(4 r^{*} / 5\right)$ lies within \pm 0.05 ; this is a satisfactory test of the accuracy achieved in the simulation). The ratio $A^{*} /\left(4 r^{*} / 5\right)$ has a maximum of about 0.49 , i.e. approximately half the value predicted by Kolmogorov (1941). Zhou \& Antonia (2000) presented results, obtained in decaying grid turbulence, for the dependence of $\left(A / r^{*}\right)$ on $R_{\lambda}$; for $R_{\lambda} \simeq 50$, figure 7 of their paper indicates a peak value of about 0.34 for $A / r^{*}$ or 0.43 for $A /\left(4 r^{*} / 5\right)$. This is in reasonable agreement with the present value; it should 

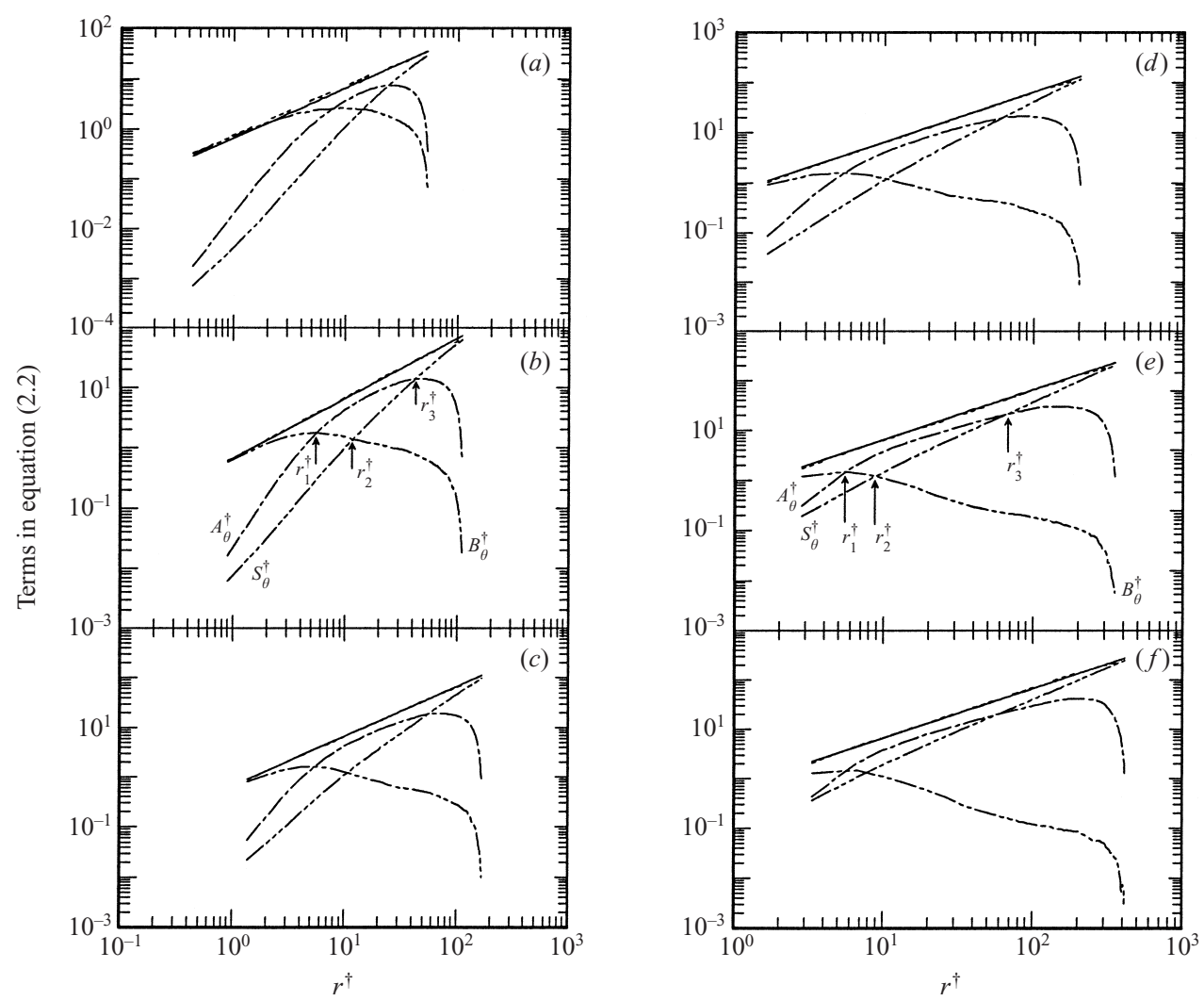

FiguRE 3. Estimates of all terms in equation (2.2), namely $A_{\theta}^{\dagger}+B_{\theta}^{\dagger}+S_{\theta}^{\dagger}=(2 / 3) r^{\dagger}$ for all values of the Schmidt number. (a) $S c=0.07$; (b) $S c=0.3$; (c) $S c=0.7$; (d) $S c=1$; (e) $S c=3$; (f) $S c=7$. $-\cdots, A_{\theta}^{\dagger} ;-\cdots, B_{\theta}^{\dagger} ;-\cdots-, S_{\theta}^{\dagger} ;-,(2 / 3) r^{\dagger} ; \cdots-\cdots, A_{\theta}^{\dagger}+B_{\theta}^{\dagger}+S_{\theta}^{\dagger}$.

be underlined however that this low value simply reflects the importance of the nonstationary term in the present study or the non-homogeneity in the experiments of Zhou \& Antonia (2000).

The terms in (2.2) are shown in figure 3 for all values of $S c$. As in figure 2, the contribution from the non-stationary term $S_{\theta}^{\dagger}$ continues to increase with $r^{\dagger}$ and becomes dominant at large $r^{\dagger}$, as required by the balance

$$
\langle\chi\rangle=-\frac{\mathrm{d}\left\langle\theta^{2}\right\rangle}{\mathrm{d} t}
$$

between the mean dissipation rate and the temporal rate of decay of the scalar variance. The non-stationarity is equally important as $r \rightarrow 0$ since, as noted in Antonia et al. (2000), the transport equation of $\langle\chi\rangle$ (as written originally by Corrsin $1953)$ is retrieved correctly at order $r^{3}$.

Irrespectively of $S c$, the relative trends of $A_{\theta}^{\dagger}, B_{\theta}^{\dagger}, S_{\theta}^{\dagger}$ are similar to those shown in figure 2. There is however an effect of $S c$ on the relative magnitudes and variations, with respect to $r^{\dagger}$, of $A_{\theta}^{\dagger}, B_{\theta}^{\dagger}$ and $S_{\theta}^{\dagger}$. For $S c=0.07$ (figure $3 a$ ), $B_{\theta}^{\dagger}$ is essentially equal to $(2 / 3) r^{\dagger}$ up to $r^{\dagger} \simeq 3$ and continues to increase to a maximum at about $r^{\dagger} \simeq 8$. This maximum corresponds approximately with the intersection $r_{1}^{\dagger}$ (see figure $3 b$ ) between $B_{\theta}^{\dagger}$ and $A_{\theta}^{\dagger}$. At larger values of $S c, r_{1}^{\dagger}$ remains essentially constant $(\simeq 6)$. The importance of $S_{\theta}^{\dagger}$ vis-à-vis $B_{\theta}^{\dagger}$ and $A_{\theta}^{\dagger}$ can be inferred from the variation with 


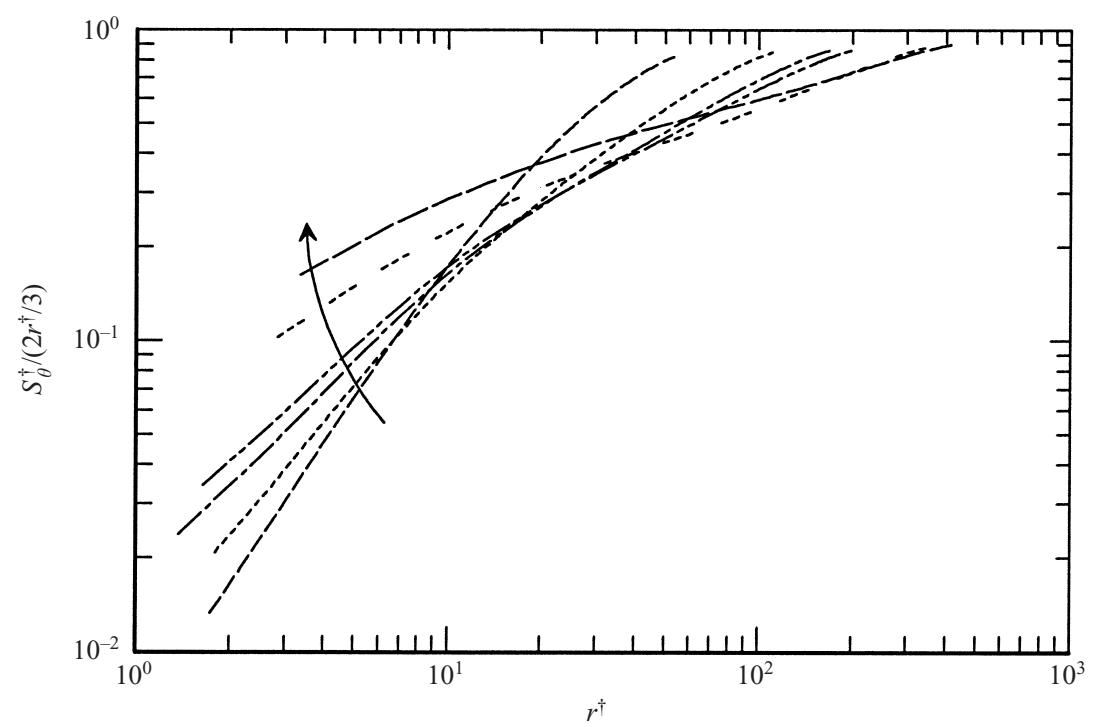

FIGURE 4. Ratio of the normalized source term $S_{\theta}^{\dagger}$ to $\left(2 r^{\dagger} / 3\right)$. The arrow is in the direction of increasing $S c$. Line types are as in figure 1.

$S c$ of $r_{2}^{\dagger}$ and $r_{3}^{\dagger}$, the intersections (identified in figure 3) of $S_{\theta}^{\dagger}$ with $B_{\theta}^{\dagger}$ and $A_{\theta}^{\dagger}$ respectively. The magnitude of $r_{2}^{\dagger}$ continues to decrease as $S c$ increases whereas $r_{3}^{\dagger}$ increases initially before becoming approximately constant $(\simeq 60)$ when $S c \gtrsim 0.7$. The accuracy with which $S_{\theta}^{\dagger}$ has been estimated can be assessed by comparing the sum $\left(A_{\theta}^{\dagger}+B_{\theta}^{\dagger}+S_{\theta}^{\dagger}\right)$ with $\left(2 r^{\dagger} / 3\right)$. Except at $S c=0.07$ (figure $\left.3 a\right)$, these two quantities are nearly indistinguishable in figure 3 . The imbalance $I_{\theta}^{\dagger}$, or difference between $\left(2 r^{\dagger} / 3\right)$ and $\left(A_{\theta}^{\dagger}+B_{\theta}^{\dagger}+S_{\theta}^{\dagger}\right)$, is largest at $S c=0.07$ (figure $3 a$ ). In this latter case, $I_{\theta}^{\dagger} /\left(2 r^{\dagger} / 3\right)$ is about -0.15 in the range $1 \lesssim r^{\dagger} \lesssim 10$. For all other values of $S c, I_{\theta}^{\dagger} /\left(2 r^{\dagger} / 3\right)$ lies within \pm 0.05 over the range $1 \lesssim r^{\dagger} \lesssim 50$. Figure 4 underlines the important overall contribution from $S_{\theta}^{\dagger}$ to the right-hand side of (2.2), irrespectively of $r^{\dagger}$. At large $r^{\dagger}$, the ratio $S_{\theta}^{\dagger} /\left(2 r^{\dagger} / 3\right)$ approaches 1 , as expected from (4.2). At small $r^{\dagger}$, there is a systematic increase with $S c$; e.g. at $r^{\dagger} \simeq 3$, the ratio increases from about $3 \%$ at $S c=0.07$ to $15 \%$ at $S c=7$.

The collapse at large $k^{\dagger}$ of the scalar spectra shown in figure 1 implies that the Batchelor-normalized second-order scalar structure functions should collapse at sufficiently small $r^{\dagger}$. It is of interest to consider the behaviour of the mixed third-order velocity-scalar structure functions at small $r^{\dagger}$. For this purpose, the ratio $A_{\theta}^{\dagger} / r^{\dagger^{3}}$ has been plotted in figure 5. The limiting value when $r \rightarrow 0$ of $D_{L \theta \theta} \equiv\left\langle\delta u(\delta \theta)^{2}\right\rangle$ is given by $\left\langle(\partial u / \partial x)(\partial \theta / \partial x)^{2}\right\rangle r^{3}$; here, $\delta u \equiv u(x+r)-u(x)$ and $\delta \theta=\theta(x+r)-\theta(x)$, with $r$ the separation in the $x$-direction. Assuming local isotropy, the limiting value of $A_{\theta}^{\dagger} / r^{\dagger^{3}}$ is $S c^{-1 / 2} S_{T} /\left(6 \times 15^{1 / 2}\right)$, where $S_{T} \equiv-\left\langle(\partial u / \partial x)(\partial \theta / \partial x)^{2}\right\rangle /\left[\left\langle(\partial u / \partial x)^{2}\right\rangle^{1 / 2}\left\langle(\partial \theta / \partial x)^{2}\right\rangle\right]$ is the so-called mixed derivative skewness (Antonia, Chambers \& Browne 1983 had earlier indicated that the limiting value of $-D_{L \theta \theta}^{* 3} / r^{* 3}$ is given by $\operatorname{Pr}_{T} /\left(6 \times 15^{1 / 2}\right)$. Sreenivasan \& Antonia (1997) noted that the magnitude of $S_{T}$ depends only weakly on $R_{\lambda}$, but the dependence of $S_{T}$ on $S c$ was unclear. The inset in figure 5 highlights the behaviour of the data as $r^{\dagger} \rightarrow 0$. For $S c=0.07,0.3,0.7$ and 1, the trend of the data suggests that the most likely value of $S_{T}$ is 0.5 (this corresponds to the dashed 


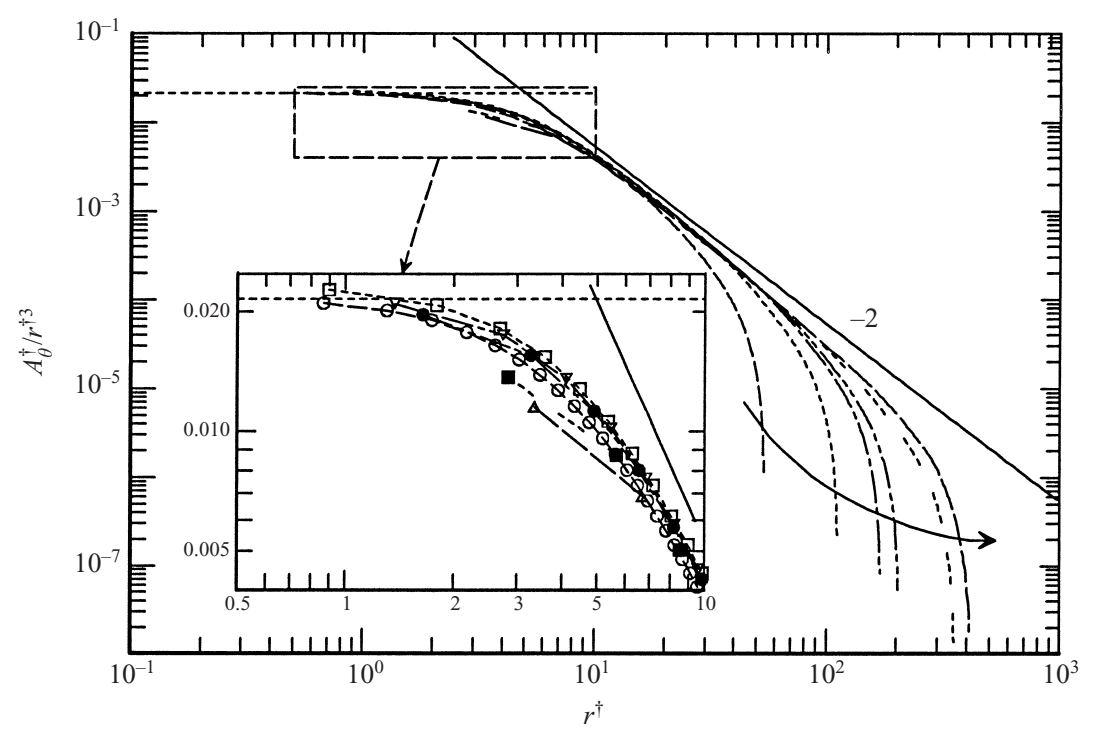

FIGURE 5. Ratio $A_{\theta}^{\dagger} / r^{\dagger^{3}}$ as a function of $r^{\dagger}$ for all values of $S c$. The solid arrow is in the direction of increasing $S c$. The horizontal dashed line corresponds to a value of $S_{T} /\left(6 \times 15^{1 / 2}\right)$, with $S_{T}=0.5$. The solid line has a slope of -2 which corresponds to a linear dependence on $r$ of $D_{L \theta \theta}$. The inset is a view of the upper left corner of the figure using larger scales. The symbols in the inset are the

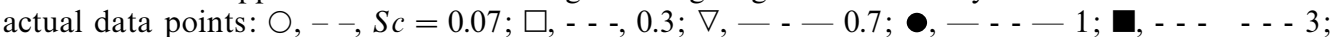
$\triangle,-, 7$.

horizontal line) with a variation of about \pm 0.03 . The results in figure 3 of Kerr (1985) for $S c=0.5,1$ and 2 (and $R_{\lambda} \gtrsim 30$ ) also indicate a value of 0.5 for $S_{T}$ with a variation comparable to that in figure 5. For $S c=0.1$, Kerr obtained a smaller value of $S_{T}$ $\left(\simeq 0.41\right.$ at $\left.R_{\lambda}=50\right)$. The results of Wang, Chen \& Brasseur (1999) for $S c=0.7$ and 1 indicated an average value of $S_{T}$ of about 0.48 . It is difficult to estimate $S_{T}$ for the present data at $S c=3$ and 7 since the first data point is at $r^{\dagger}=2.84$ for $S c=3$ and at 3.35 for $S c=7$. The resolution for these two runs is not sufficient to allow a reliable estimate of $S_{T}$. The difficulty of achieving reliable estimates of $S_{T}$ when $S c$ exceeds 1 has been discussed in some detail in Orlandi \& Antonia (2001). A likely source of error is the non-closure of the integrand in $\int_{0}^{\infty} k_{1}^{4} \phi_{\theta}\left(k_{1}\right) \mathrm{d} k_{1}$, where $\phi_{\theta}\left(k_{1}\right)$ is the one-dimensional scalar spectrum ( $k_{1}$ is the one-dimensional wavenumber). For the $384^{3}$ pseudo-spectral simulation at $S c=7$ (see figure 1), we inferred a value of about 0.49 for $S_{T}$ using the previous integral. Although this is an indirect estimate based on the assumption that $\left\langle(\partial u / \partial x)(\partial \theta / \partial x)^{2}\right\rangle$ is equal to $-(2 / 3)\left\langle\left(\partial^{2} \theta / \partial x^{2}\right)^{2}\right\rangle$, it does not rule out a possible approach towards $S_{T} \simeq 0.5$ for the present distributions at $S c=3$ and 7. Further, more refined, simulations are needed to allow a more definite statement regarding the independence of $S_{T}$ from $S c$.

\section{Conclusions}

DNS data for temporally decaying quasi-isotropic turbulence confirm the importance of the non-stationarity in the generalized form of Kolmogorov's equation, (2.1), as previously established for spatially decaying inhomogeneous turbulence downstream of a grid by Danaila et al. (1999) . The non-stationary term in the generalized version of Yaglom's equation, (2.2), which describes the decay of a passive scalar in 
the same flow, is equally important, irrespectively of the Schmidt number $S c$ and $r^{\dagger}$. The importance, when varying $S c$, of the terms $A_{\theta}^{\dagger}, B_{\theta}^{\dagger}$ and $S_{\theta}^{\dagger}$ in (2.2) can be gauged from figures 3 and 4 . The latter figure highlights the increased importance of the non-stationarity at small $r^{\dagger}$, as $S c$ increases. The present results also confirm the appropriateness of normalizing with Batchelor scales at small separations, at least when $S c$ is not too small. In particular, there is reasonable collapse over the range $r \lesssim 20 \eta_{B}$ of the third-order term $A_{\theta}^{\dagger}$. The trend of $A_{\theta}^{\dagger} / r^{\dagger^{3}}$ (figure 5) at small $r^{\dagger}$ implies that the magnitude of the mixed velocity-scalar derivative skewness is constant $(\simeq 0.5 \pm 0.03)$ for $0.07 \leqslant S c \leqslant 1$. The trend of the data for $S c=3$ and 7 does not rule out the possibility that a value of $S_{T}$ equal to about 0.5 may also apply at these two higher Schmidt numbers. However, more work is required to verify this possibility.

We gratefully acknowledge the support of the Australian Research Council, including the allocation of a collaborative IREX grant between the University of Newcastle and La Sapienza, and a MURST 60\% grant.

\section{REFERENCES}

Alvelius, K. 1999 Random forcing of three-dimensional homogeneous turbulence. Phys. Fluids 11 $1880-1889$.

Alvelius, K. \& Johansson, A. V. 2000 LES computations and comparison with Kolmogorov theory for two-point pressure-velocity correlations and structure functions for globally anisotropic turbulence. J. Fluid Mech. 403, 23-36.

Antonia, R. A., Chambers, A. J. \& Browne, L. W. B. 1983 Relations between structure functions of velocity and temperature in a turbulent jet. Exps. Fluids 1, 213-219.

Antonia, R. A., Zhou, T., Danaila, L. \& Anselmet, F. 2000 Streamwise inhomogeneity of decaying grid turbulence. Phys. Fluids 1, 3086-3089.

BATCHELOR, G. K. 1959 Small scale variation of convected quantities like temperature in a turbulent fluid. J. Fluid Mech. 5, 113-133.

Batchelor, G. K., Howells, I. D. \& Townsend, A. A. 1959 Small scale variation of convected quantities like temperature in turbulent fluid. Part 2. The case of large conductivity. J. Fluid Mech. 5, 134-139.

Batchelor, G. K. \& Townsend, A. A. 1947 Decay of vorticity in isotropic turbulence. Proc. R. Soc. Lond. A 190, 534-550.

Bogucki, D., Domaradzki, J. A. \& Yeung, P. K. 1997 Direct numerical simulations of passive scalars with $\operatorname{Pr}>1$ advected by turbulent flow. J. Fluid Mech. 343, 111-130.

Brethouwer, G. \& Nieuwstadt, F. T. M. 1999 Mixing of weakly and strongly diffusive scalars in isotropic turbulence. In Direct and Large-eddy Simulation III, Proc. Isaac Newton Institute Symposium/ERCOFTAC Workshop, Cambridge, p. 311. Kluwer.

Corrsin, S. 1951 The decay of isotropic temperature fluctuations in an isotropic turbulence. J. Aero. Sci. 18, 417-423.

Corrsin, S. 1953 Remarks on turbulent heat transfer: an account of some features of the phenomenon in fully turbulent regions. In Proc. Iowa Thermodynamics Symposium, pp. 5-30. State University of Iowa.

Danaila, L., Anselmet, F., Zhou, T. \& Antonia, R. A. 1999 A generalization of Yaglom's equation which accounts for the large-scale forcing in heated grid turbulence. J. Fluid Mech. 391, 359-372.

Danaila, L., Zhou, T., Anselmet, F. \& Antonia, R. A. 2000 Calibration of a temperature dissipation probe in decaying grid turbulence, Exps. Fluids 28, 45-50.

Gibson, C. H. 1968 a Fine structure of scalar fields mixed by turbulence. I. Zero-gradient points and minimal gradient surfaces. Phys. Fluids 11, 2305-2315.

Gibson, C. H. 1968 b Fine structure of scalar fields mixed by turbulence. II. Spectral theory. Phys. Fluids 11, 2316-2327.

Gibson, C. H. 1991 Kolmogorov similarity hypotheses for scalar fields: sampling intermittent turbulent mixing in the ocean and galaxy. Proc. R. Soc. Lond. A 434, 149-164. 
HiLl, R. J. 2001 Equations relating structure functions of all orders. J. Fluid Mech. 434, 379-388.

von KÁrmán, T. \& Howarth, L. 1938 On the statistical theory of isotropic turbulence. Proc. R. Soc. Lond. A 164, 192-215.

Kerr, R. M. 1985 Higher-order derivative correlations and the alignment of small-scale structures in isotropic numerical turbulence. J. Fluid Mech. 163, 31-58.

KerR, R. M. 1990 Velocity, scalar and transfer spectra in numerical turbulence. J. Fluid Mech. 211, 309-332.

Kolmogorov, A. N. 1941 Dissipation of energy in locally isotropic turbulence. Dokl. Akad. Nauk. SSSR 32, 16-18.

LiNDBORG, E. 1999 Correction to the four-fifths law due to variations of the dissipation. Phys. Fluids 11, 510 .

Monin, A. S. \& Yaglom, A. M. 1975 Statistical Fluid Mechanics, Vol. 2. MIT Press.

Nieuwstadt, F. T. M. \& Brethouwer, G. 2000 Turbulent transport and mixing. In Advances in Turbulence VIII (ed. C. Dopazo), pp. 133-140. Barcelona, CIMNE.

Orlandi, P. \& ANTONia, R. A. 2001 Direct numerical simulations of decaying turbulent passive scalar fluctuations: Schmidt number dependence. J. Fluid Mech. (submitted).

Pumir, A. 1994 Small-scale properties of scalar and velocity differences in three-dimensional turbulence. Phys. Fluids 6, 3974-3984.

Saffman, P. G. 1968 Lectures on homogeneous turbulence. In Topics in Non-linear Physics (ed. N. J. Zabusky), pp. 485-614. Springer.

Sreenivasan, K. R. \& Anontia, R. A. 1997 The phenomenology of small-scale turbulence. Annu. Rev. Fluid Mech. 29, 435-472.

Wang, L.-P., Chen, S. \& Brasseur, G. J. 1999 Examination of hypotheses in the Kolmogorov refined turbulence theory through high-resolution simulations. Part 2. Passive scalar field. J. Fluid Mech. 400, 163-197.

Warhaft, Z. 2000 Passive scalars in turbulent flows. Annu. Rev. Fluid Mech. 32, 203-240.

YAGLOM, A. M. 1949 On the local structure of a temperature field in a turbulent flow. Dokl, Akad, Nauk, SSSR 69, 743-746.

Yeung, P. K., Sykes, M. C. \& Vedula, P. 2000 Direct numerical simulation of differential diffusion with Schmidt numbers up to 4.0. Phys. Fluids 12, 1601-1604.

Zhou, T. \& Antonia, R. A. 2000 Reynolds number dependence of the small-scale structure of grid turbulence. J. Fluid Mech. 406, 81-107.

Zhou, T., Antonia, R. A., Danaila, L. \& Anselmet, F. 2000 Transport equations for the mean energy and temperature dissipation rates in grid turbulence. Exps. Fluids 28, 143-151. 